\title{
DESARROLLO DE UNA CONCIENCIA AMBIENTAL EN UNA PROPUESTA DE INTERCOMPRENSIÓN EN LENGUAS ROMANCES PARA ESTUDIANTES DE NIVEL MEDIO
}

\author{
Bibiana AMADO* \\ Ana Cecilia PÉREZ** \\ Richard Brunel MATIAS***
}

- RESUMEN: El presente trabajo analiza el tratamiento de temas ambientales en una propuesta de Intercomprensión en Lenguas Romances (IC) que el equipo de InterRom, de la Universidad Nacional de Córdoba, ha elaborado para el desarrollo de las capacidades de lenguaje de estudiantes de los últimos años de la escuela secundaria desde la óptica de la enseńanza y el aprendizaje de IC en torno a los géneros de textos. Esta propuesta integra los enfoques plurales de las lenguas con aportes del interaccionismo sociodiscursivo (BRONCKART, 2007), de la teoría sociocultural del desarrollo humano (BRUNER, 1994; NELSON, 1996; ROGOFF, 1993; VYGOTSKY, 1962, 1988) y de los estudios cognitivos sobre la comprensión y la producción de textos (BRITTON; GRAESSER, 1996; HAYES; FLOWERS, 1980, HAYES, 1996; SCARDAMALIA; BEREITER, 1992). En la convergencia de esas perspectivas teóricas, la propuesta promueve acciones de intercomprensión en francés, portugués, italiano y español, relacionadas, con la conservación del ambiente. Las actividades incluyen el trabajo con diferentes géneros textuales que abordan problemáticas ambientales. En relación con estos temas se propone la realización de actividades que promueven, al mismo tiempo, la reflexión sobre temas ambientales críticos y el desarrollo de estrategias de producción de textos escritos en español. En ese sentido, es posible sostener que la propuesta se funda en un enfoque social e intercultural.

- PALABRAS CLAVE: Plurilingüismo. Intercomprensión. Géneros textuales. Medio ambiente.

\footnotetext{
* UNC - Universidad Nacional de Córdoba. Facultad de Lenguas. Córdoba - Argentina. 5000 bibyamado@gmail.com

** UNC - Universidad Nacional de Córdoba. Facultad de Lenguas. Córdoba - Argentina. 5000 anyacecilya@gmail.com
}

*** UNC - Universidad Nacional de Córdoba. Facultad de Lenguas - Sesión Portugués. Córdoba Argentina. 5000 - richardbrunelmatias@gmail.com 


\section{Introducción}

El propósito de este trabajo es analizar las secuencias didácticas que abordan temas ambientales en el marco de una propuesta que ha elaborado el equipo de InterRom (Intercomprensión en Lenguas Romances), de la Facultad de Lenguas, de la Universidad Nacional de Córdoba, Argentina, para promover el aprendizaje de las lenguas desde los enfoques plurales en el nivel medio. Dicha propuesta integra estos enfoques con las contribuciones del interaccionismo sociodiscursivo (BRONCKART, 2007), de la teoría sociocultural del desarrollo humano (BRUNER, 1994; NELSON, 1996; ROGOFF, 1993; VYGOTSKY, 1962, 1988) y de los estudios cognitivos sobre la comprensión y la producción de textos (BRITTON; GRAESSER, 1996; HAYES; FLOWERS, 1980, HAYES, 1996; SCARDAMALIA; BEREITER, 1992). En la convergencia de esas concepciones teóricas, la propuesta no solo promueve estrategias de intercomprensión en francés, portugués, italiano y español, sino que también pone de relevancia los aprendizajes vinculados con diferentes saberes disciplinares. En el presente artículo abordaremos, en particular, el tratamiento de los temas ambientales que forman parte de los estudios en ciencias naturales. Desde nuestra perspectiva, los conceptos de las ciencias permiten crear un contexto cognitivo y dan significación social al aprendizaje de las lenguas.

En la actualidad los intercambios y las interacciones entre personas de distintas edades y culturas se han intensificado dado el uso masivo de las TIC (Tecnologías de la Información y la Comunicación). En general, los estudiantes jóvenes son los usuarios más habituados ala comunicación en las redes sociales. Sin duda, en ese contexto las lenguas juegan un papel preponderante, puesto que son la puerta de acceso para el contacto con las personas y culturas con las cuales interactuamos. De hecho, nuestros estudiantes suelen comunicarse no solo con hablantes de español, sino también de portugués, italiano, francés, inglés, entre otros idiomas, y, de una forma u otra, emplean estrategias de intercomprensión (IC). Esta posibilidad no se limita a los intercambios virtuales sino que se extiende a otras prácticas comunicativas, como la lectura de revistas, diarios, libros en diferentes lenguas. Además, nuestros estudiantes no solo leen, también suelen escribir en las redes sociales y lo pueden hacer en distintas lenguas, según sus competencias discursivas.

Ante este fenómeno resulta paradójico reconocer las dificultades que esos mismos jóvenes manifiestan en la lectura y escritura de textos incluso al finalizar los estudios secundarios. Este problema, que ha sido motivo de investigaciones en las últimas dos décadas, presenta diversas causas, entre las cuales, no se puede soslayar la ausencia de políticas educativas que tiendan a promover una educación lingüística, centrada en el desarrollo de la lectura y la escritura, desde edades tempranas hasta la adolescencia y que continúe en los estudios superiores de modo integrado con los contenidos disciplinares específicos de cada ciencia. Atendiendo a la adquisición de las lenguas extranjeras, es posible considerar que las metodologías de enseñanza y de 
aprendizaje que predominan en nuestro país desde hace algunas décadas conciben los aprendizajes lingüísticos en forma aislada, no tienden a una enseñanza integrada de lenguas, propia de los enfoques plurales y, con ello, no favorecen la interrelación y la reflexión metalingüística.

Frente a esta problemática, desde el año 2000, los profesores e investigadores del equipo InterRom hemos promovido en el ámbito universitario y, más recientemente, en la escuela secundaria el trabajo con propuestas pedagógicas para una educación en lenguas romances que promueven la recuperación de los espacios curriculares para la diversidad lingüístico-cultural y el desarrollo de la competencia discursiva en la propia lengua materna.

Nuestro proyecto actual, denominado Enfoques Plurales en la Escuela Secundaria. Impactos de la intercomprensión en lenguas romances en el desarrollo de las competencias lingüistico-discursiva e intercultural en adolescentes escolarizados, avalado por la SecytUNC (Secretaría de Ciencia y Técnica de la Universidad Nacional de Córdoba), pretende dar continuidad a la línea de investigación desarrollada por el equipo InterRom en el campo de la didáctica del plurilingüismo. De esta manera, estamos promoviendo el conocimiento de las lenguas desde un enfoque intercomprensivo, que haga posible el desarrollo de una competencia plurilingüe y pluricultural, integrando los aprendizajes lingüísticos con los de otras áreas disciplinares.

Con el proyecto de investigación y de intervención en el nivel medio, iniciado en 2012, estamos dando los primeros pasos hacia la incorporación de la perspectiva de los enfoques plurales en enseñanza de lenguas extranjeras en el sistema educativo. También estamos propiciando la formación de docentes de lenguas que ayuden a formar ciudadanos responsables, críticos, que tengan la capacidad de asumir el rol de mediadores interculturales, que fomenten el desarrollo de una competencia de comunicación que promueva encuentro con el otro en la diversidad.

Es nuestra expectativa que los jóvenes no se limiten a usar las redes sociales solo como medio para practicar una nueva lengua o buscar contactos, desde una visión estrictamente instrumental, sino que sean actores sociales plurilingües y pluriculturales, personas que fomenten actitudes positivas, de respeto hacia la diversidad lingüística y cultural. Por otro lado, mediante nuestra propuesta didáctica podrían desarrollar sus capacidades de lenguaje dado que, desde nuestra perspectiva, la adquisición de habilidades intercomprensivas en francés, italiano y portugués vinculadas con los saberes disciplinares, contribuye al aprendizaje de la escritura de textos en español lengua materna.

\section{Una aproximación social a los temas ambientales en la educación lingüística}

Así como remarcamos la importancia de la educación en lenguas también entendemos que el tema ambiental integra la agenda de los problemas sociales más relevantes de la 
sociedad contemporánea y, por ello, debe formar parte de los contenidos transversales de la educación obligatoria. Incorporar contenidos ambientales a los espacios curriculares de lenguas (materna y extranjeras) significa reconocer el valor formativo que ofrece el tratamiento del ambiente, pues involucra una serie de aspectos que consideramos clave en la construcción del conocimiento científico, la formación ética, la actuación ciudadana crítica y responsable de los jóvenes y garantizan el cumplimiento de uno de los propósitos de la educación secundaria. En tal sentido, el artículo 30, inciso a) de la Ley de Educación Nacional 26.206 de Argentina (2006), señala como objetivo:

Brindar una formación ética que permita a los/as estudiantes desempeñarse como sujetos conscientes de sus derechos y obligaciones, que practican el pluralismo, la cooperación y la solidaridad, que respetan los derechos humanos, rechazan todo tipo de discriminación, se preparan para el ejercicio de la ciudadanía democrática y preservan el patrimonio natural y cultural.

Construir una propuesta de enseñanza en torno del ambiente nos obliga, como educadores, a asumir una perspectiva, o al menos a acordar criterios, en el tratamiento de aspectos ambientales. Si asumimos, tal como lo plantea Gurevich (2011, p.18), la cuestión ambiental "[...] como la expresión compleja y contradictoria de las acciones de sujetos sociales concretos en determinadas coordenadas históricas [...]", convenimos en abordar estas manifestaciones, complejas y contradictorias, desde una dimensión social que considera las relaciones entre naturaleza y sociedad recíprocamente condicionadas.

Desde esta concepción de ambiente como una categoría relacional, el enfoque de los temas incluidos en las secuencias didácticas de nuestra propuesta, toma en cuenta el sistema de valores, de saberes y conductas de las comunidades involucradas así como los modos en los que cada grupo social se compromete con su ambiente. Para abordar la dimensión socio-ambiental, en nuestro proyecto consideramos la idea de ambiente como una construcción colectiva, culturalmente situada que, en la toma de decisiones conjuntas, integra aspectos de la sociedad, la cultura, la política y la economía, entre otros. Estos grandes principios han orientado la selección de aspectos vinculados con el uso y el manejo de los recursos naturales, eje en el que confluye un abanico de temas que posibilitan acercarnos a la complejidad de las problemáticas ambientales.

\section{Los textos sobre el ambiente y las estrategias de intercomprensión}

La intercomprensión integra los llamados enfoques plurales de las lenguas y de las culturas (CANDELIER, 2008) relacionados con las perspectivas didácticas que incluyen las variedades lingüísticas y culturales y se oponen a las concepciones centradas en una lengua y en una cultura de modo aislado, como sucede con los enfoques singulares. En nuestra propuesta de enseñanza, la intercomprensión entre lenguas de la familia románica se orienta al desarrollo de estrategias de comprensión de géneros textuales 
en cuatro lenguas romances (portugués, francés, italiano, español) en el marco de un sistema de actividades que tiene en cuenta la interdependencia entre los procesos de lectura y escritura.

En este sentido, y con el fin de orientar el proceso de escritura en lengua materna sobre temas ambientales, las situaciones de lectura cumplen un rol fundamental, en tanto instancias de trabajo que posibilitan recuperar conocimientos previos y experiencias, facilitar la generación de ideas en torno a temas relacionados al ambiente y construir una especie de archivo con informaciones y conceptos que estén disponibles en el momento de construcción de los textos.

La propuesta de actividades se diseñó a partir de una selección de géneros textuales, en las cuatro lenguas de trabajo, que incluye: artículos enciclopédicos, leyendas y notas de opinión. Los criterios de selección de estos recursos, además de la diversidad de géneros y soportes y del grado de intercomprensión, determinado por la presencia de léxico y estructuras sintácticas panrománicas, consideran la referencia a una escena socio-ambiental que destaca la relación entre la vida social, económica, política con la dinámica ambiental de un grupo cultural en un momento histórico determinado.

A continuación analizaremos nuestra propuesta de trabajo con temas ambientales desde tres dimensiones relacionadas con el proceso de elaboración del material didáctico. Dichas dimensiones exploran los modos de significar el ambiente, los modos de entender la conservación del ambiente y el carácter mediador del lenguaje en los textos vinculados con esta problemática.

\section{Modos de significar el ambiente}

Distintos investigadores sostienen que en las últimas décadas se registra la mayor tasa de extinción de especies animales y vegetales (CURTIS et al., 2011). En diversas regiones de América, el yaguareté (onça pintada) representa una especie que está en estado crítico de extinción, entre otras causas, debido a la destrucción de su hábitat. Por ello, Argentina lo ha declarado Monumento Natural de la Nación y su imagen representa el símbolo de la preservación del medio natural (CANEVARI; FERNÁNDEZ BALBOA, 2003). En nuestra propuesta de enseńanza, este felino es enfocado en las dos primeras secuencias didácticas, en las cuales abordamos el artículo enciclopédico y la leyenda. De este modo, el tema del yaguareté es presentado en dos géneros textuales distintos, lo que establece diferencias no solo en el carácter de la información sino también en las perspectivas de la enunciación y en los modos de organizar el contenido de los textos.

Si entendemos, desde una concepción bajtiniana, que los géneros discursivos configuran series de enunciados que mediatizan prácticas sociales, podemos sostener que el artículo enciclopédico y la leyenda representan dos vías de aproximación al conocimiento en esferas diferenciadas de la praxis social. En gran medida, esta idea se corresponde con la noción de formas de ejercicio de la racionalidad, que Gasché (2004) emplea para explicar los modos de comprender los fenómenos naturales que 
manifiestan diversas etnias de la amazonia peruana. Así pues, mientras el artículo enciclopédico emplea los recursos enunciativos de la prosa científica, en la leyenda subyace un sistema explicativo diferente, donde ciertos fenómenos naturales son explicados a partir de eventos sobrenaturales.

En la primera secuencia didáctica, se propone la lectura de artículos enciclopédicos en italiano, francés, portugués y español. Estos textos posibilitan el acceso a diversos conceptos científicos sobre el yaguareté, vinculados con el parentesco con otras especies, sus métodos de caza, alimentación, reproducción y características físicas. En los cuatro textos predominan secuencias explicativas y descriptivas (ADAM, 1996); no obstante, cada uno de ellos presenta perspectivas diferenciadas y complementarias sobre el yaguareté y su entorno (Figura 1).

Figura 1 - Esquema que muestra los temas

desarrollados en cada texto sobre el yaguareté

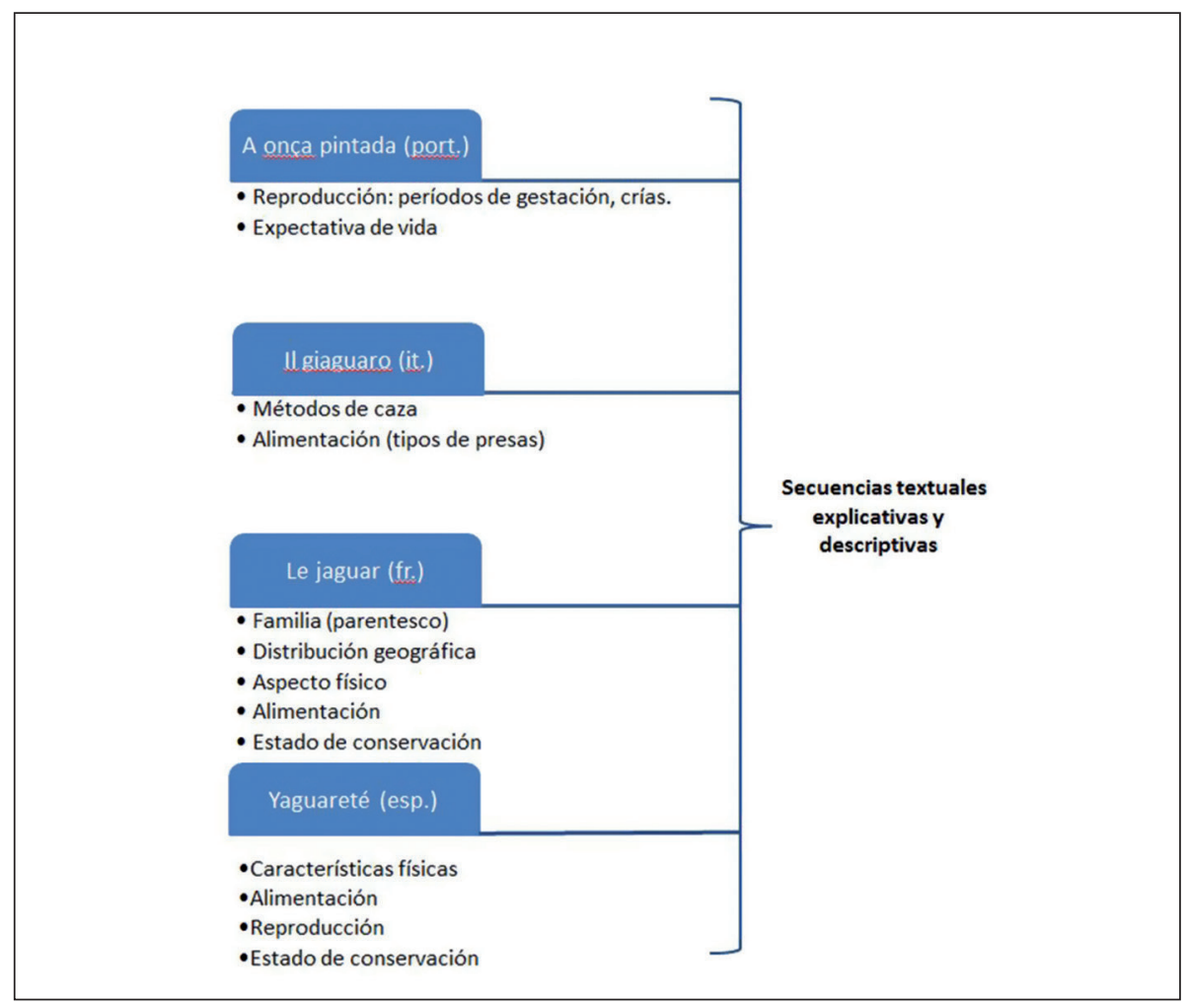

Fuente: Elaboración propia.

La lectura de la información proporcionada en los cuatro idiomas permite conformar una base de conocimientos completa sobre la especie estudiada. Por ejemplo, mientras 
el texto en italiano y portugués centran el interés en aspectos propios de la especie, el texto en francés pone en evidencia el riesgo que afecta a este felino por la acción del hombre. Es, precisamente, este último texto el que abre la posibilidad de profundizar en el estado de conservación y aporta una visión más integral del ambiente al enfocar los vínculos entre la continuidad de la especie y la actividad humana.

En la segunda secuencia proponemos la lectura de "El jaguar y el fuego", un relato escrito por Eduardo Galeano a partir de una leyenda de la comunidad Kayapó, que habita en Brasil, en el sur de la Amazonia. En la versión de Galeano, el yaguareté, que cazaba con arco y flecha, encuentra a un joven kayapó que estaba hambriento y lo invita a su casa. Allí, el joven conoce el fuego y, por primera vez, come carne asada. Sin embargo, no es recibido de buena manera por la mujer del jaguar. El joven aprende a manejar el arco y un día mata a aquella mujer. Entonces huye hasta su comunidad y cuenta a su gente sobre la existencia del fuego y las armas del jaguar. Los kayapó quieren apoderarse de ambos elementos, por eso el joven los guía hasta la casa de su protector y ellos roban el fuego y las armas. Desde entonces el jaguar odia a los hombres, que lo dejaron sin el arco y las flechas. Del fuego, solo le quedó el brillo reflejado en sus ojos.

Los hechos de la leyenda permiten explicar, en particular, cómo el ser humano conoció el fuego, por qué el yaguareté "odia” al hombre y por qué brillan los ojos de ese felino. Atenderemos a esta última explicación con el fin de confrontar la perspectiva de la leyenda con la del artículo enciclopédico, pues en uno y otro género subyacen dos formas de ejercicio de la racionalidad y, por ende, dos modos diferenciados de significar el ambiente.

De acuerdo con la leyenda, el brillo de los ojos del yaguareté se debe al reflejo del fuego, que los seres humanos robaron a ese felino. Esta explicación se basa en un relato, una secuencia de hechos que tuvo, entre otras consecuencias, esa modificación en los ojos. Desde la perspectiva de la ciencia, este fenómeno, propio de los felinos y de otras especies cazadoras, se explica de un modo muy diferente: los ojos de estos animales tienen, detrás de la retina, una membrana compuesta de células reflectantes, denominada tapetum lucidum, que refleja los rayos luminosos. Esa membrana permite intensificar la visión en condiciones de escasa luminosidad y, al mismo tiempo, hace que los ojos brillen en la penumbra.

Ambas formas de explicar los fenómenos del mundo natural, que coexisten en la especie humana, hacen referencia a diferentes modos de ejercicio de la racionalidad. Desde una perspectiva pedagógica, es sumamente necesario que los alumnos no solo conozcan los sistemas explicativos que operan en uno y otro género textual sino que reflexionen sobre dichos sistemas para comprender la funcionalidad que tienen en cada contexto de producción. Pues, así como para algunos grupos culturales la explicación mítica del relato del jaguar es suficiente, en otros entornos, es preciso conocer las causas que aporta la ciencia. Sin duda, en estos últimos entornos culturales, la leyenda ocupa un espacio diferente como género textual. Es decir, el lector de la leyenda entenderá 
el contenido no como una explicación del mundo natural, sino como una explicación dentro del contexto narrativo.

Para nuestro equipo es un verdadero desafío poner en diálogo estos géneros textuales como la leyenda y el artículo enciclopédico, en un material didáctico cuyo propósito no es "prescribir" cómo "debe ser" un género, sino describir funciones y procedimientos discursivos y, al mismo tiempo, proporcionar oportunidades para que los estudiantes disfruten de la lectura de una leyenda y conozcan los modos de explicación y las posibilidades de enunciación que proporciona cada género. De ese modo, no proponemos el discurso de la ciencia como una verdad única y la leyenda como un mero entretenimiento sino que nuestro objetivo es que los alumnos conozcan diferentes conceptos y los procedimientos de cada modelo textual. Al mismo tiempo, es nuestra expectativa que los estudiantes comprendan los patrones de pensamiento que propone cada forma discursiva y, con ello, que puedan problematizar la idea de un "pensamiento único" y entender que la mente humana puede ejercer, de acuerdo con las culturas, diferentes modalidades para significar la realidad.

\section{Modos de entender la conservación del ambiente}

Como se sabe, desde hace varias décadas ciertos grupos con conciencia por el medio natural comenzaron a preocuparse por la conservación del ambiente, en especial, a partir del deterioro producido por los procesos de industrialización y el empleo de recursos tecnológicos que son nocivos para la biosfera. Como respuesta a los problemas ambientales se han propuesto diferentes acciones para preservar los recursos de la naturaleza. Básicamente, citaremos dos perspectivas, una, que promueve un uso racional, más conservador, de dichos recursos y otra, que plantea un empleo sustentable, que considera las necesidades de los grupos humanos, entendiendo que el uso de los recursos debe contemplar procesos de planificación y consenso (CURTIS et al., 2011).

Por otra parte, la preocupación por el cuidado del ambiente ha sido enfocada de diferente forma en los materiales de estudios para el nivel secundario, donde muchas veces se simplifica esta problemática o bien se la presenta como un fenómeno alejado de la realidad cotidiana de los estudiantes. De hecho, el abordaje de los problemas ambientales representa un desafío en el ámbito escolar, entre otros motivos, debido a que la misma idea de conservación es compleja, en particular, cuando se incluyen las sociedades humanas en la noción de ambiente. En la elaboración de nuestro material didáctico tomamos como referencia el concepto que propone la Unión Internacional para la Conservación de la Naturaleza y Recursos Naturales (UICN), que define la conservación como "[...] la utilización humana de la biosfera para que rinda el máximo beneficio sostenible, a la vez que mantiene el potencial necesario para las aspiraciones de futuras generaciones." (AGUILAR et al., 2006). 
Esa definición nos plantea una pregunta básica que requiere ser atendida en las situaciones de enseñanza: ¿Qué significa "mantener el potencial de la biosfera" para que las generaciones futuras puedan hacer uso de los recursos naturales? A partir de este interrogante, en la tercera secuencia didáctica de nuestra propuesta enfocamos la problemática de la conservación del ambiente a partir de situaciones actuales que son críticas en nuestro país - Argentina- y en nuestro continente. Para mantener la continuidad temática entre las tres secuencias, en primer término abordamos el conflicto en el que está involucrada la comunidad kayapó por la construcción de represas hidroeléctricas en el Río Xingu, en la Amazonia brasilera. Para conocer y reflexionar sobre la conservación del ambiente, enfocamos el artículo de opinión, como forma textual pertinente en tanto promueve el debate, la confrontación de opiniones y la necesidad de asumir una postura respecto de los problemas abordados.

El enfoque de dicha problemática se integra en el contexto cognitivo que crea la leyenda del yaguareté, de origen kayapó, y, además, nos permite profundizar conocimientos sobre esa etnia. En este sentido, es preciso destacar que muchas veces en los materiales didácticos las referencias a las culturas originarias de nuestro continente se limitan al tiempo pasado o bien al espacio de la ficción. Abordar un conflicto actual de la comunidad kayapó permite atender el protagonismo social de estos grupos culturales y, al mismo tiempo, comprender la complejidad que entraña la conservación del ambiente, pues no solo estamos atendiendo a la idea de cuidado de la biodiversidad de las especies, sino al hecho de que la preservación de la biosfera supone necesariamente mantener la diversidad cultural atendiendo las necesidades de todos los grupos humanos que la habitan.

Figura 2 - Noticia sobre luchas actuales de la etnia Kayapó

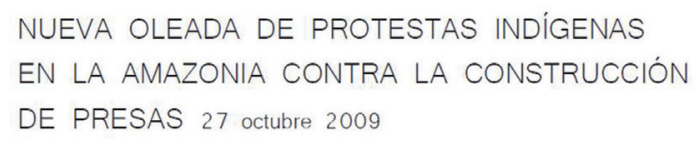

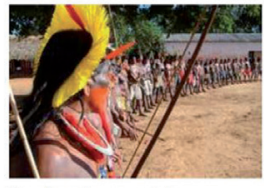

Danza kayapó en una protesta anti presas, $2006 \odot \mathrm{T}$ Turner o T Turner espera que acudan unos 200 indigenas.

Representantes del Ministerio de Minas y Energía de Brasil y el Ministro del Ambiente han sido invitados para hablar con los indígenas. Los kayapó y otros pueblos indígenas se oponen a la presa, argumentando que no se les ha consultado de forma adecuada y que no han sido informados acerca del verdadero impacto que tendrá sobre sus tierras.

Fuente: http://www.survival.es/noticias/5163

Fuente: SURVIVAL (2009). 
Figura 3 - Características de la etnia Kayapó

\begin{abstract}
Oggi, in Brasile, distribuiti in circa 225 gruppi, vivono poco più di 350.000 indios. Erano alcuni milioni prima della conquista da parte degli Europei. Nei circa 5 secoli trascorsi da quel tragico evento, alcune centinaia di questi gruppi sono scomparsi per sempre.

Tra le popolazioni sopravvissute, vi sono i KAYAPO'. Abitano la foresta amazzonica, in un territorio grande più di un quarto dell'Italia. "Kayapò" è $i 1$ nome che altri hanno loro attribuito. Essi, da parte loro, si autodefiniscono "Mebêngôkre": "il popolo che venne dall'acqua". Vivono dispersi in 17 villaggi, ognuno dei quali è un'entità a sé. Costruiti in zone isolate, i villaggi Kayapò presentano, normalmente, una struttura circolare, con in mezzo la casa degli uomini, centro propulsore ed organizzativo di tutta la vita del villaggio. Il mito racconta che questo modello circolare del villaggio è stato copiato dalle arnie delle api selvatiche. I Kayapò danno grande importanza alle diverse feste durante le quali rivivono, nel rito della danza e della gioia, il tempo degli antenati e della loro nascita come popolo.

http://www.nonsolonoi.e-cremona.it/kayapo/kayapo.htm
\end{abstract}

Fuente: Corradini (2003).

Además del conflicto de la comunidad kayapó, proponemos el trabajo con otros textos argumentativos que presentan diferentes perspectivas en torno de la explotación de zonas agrícolas en Argentina, donde la intervención humana en los sistemas ambientales está provocando el deterioro del medio natural, de las especies que lo habitan y de la calidad de la vida humana. La tendencia de las prácticas agrícolas en los últimos años ha generado la disminución de la diversidad en los cultivos, la eliminación de áreas naturales, que, entre otras trasformaciones, se suman al empleo de agroquímicos que afectan al ser humano y a otras especies de la biosfera. La información sobre los desmontes para el cultivo de la soja, la instalación de multinacionales que promueve prácticas de cuidado de los cultivos que son nocivas para el ambiente y la lucha de las organizaciones no gubernamentales contra estos intereses son algunos de los temas abordados en la tercera secuencia didáctica. Estos fenómenos, que también acontecen en la provincia de Córdoba (Argentina), son presentados en nuestro material didáctico con el propósito de que los estudiantes reflexiones sobre las cuestiones del ambiente próximas a nuestra vida cotidiana.

\title{
La mediación del lenguaje
}

Desde la perspectiva de Vygotsky $(1962,1988)$, el lenguaje es reconocido como una herramienta esencial en el desarrollo de la cognición. En consonancia con esta idea, Halliday (1993) sostiene que la experiencia deviene conocimiento mediante el lenguaje, mientras que Bajtín (1982) enfatiza en los géneros discursivos como formas de mediación semiótica (SILVESTRI, 2000). Como se sabe, Bajtín plantea que, en distintas esferas de la actividad humana la comunicación está mediada por géneros discursivos, formas relativamente estables de enunciados que presentan rasgos comunes, como los modos de organizar la información, los estilos vinculados con el 
ámbito social donde se producen, los procedimientos para estructurar la información y la relación con el destinatario asociada a la intención del discurso.

Debido a que estas formas genéricas se configuran a partir de la necesidad de comunicación propia de una esfera de la praxis social, para lograr intercomprensión es necesario que los hablantes no solo dominen los componentes léxicos y gramaticales de una lengua, sino también los géneros discursivos que median la información (SILVESTRI, 1994). De igual modo, tanto la enseñanza de lengua materna como de lenguas extranjeras no puede pensarse desvinculada de los conceptos que se construyen en los distintos ámbitos sociales donde se hablan esas lenguas.

Por ello, en nuestra propuesta de enseñanza los temas sobre el ambiente dan unidad al estudio de los contextos de enunciación, de distintas formas discursivas y de los mecanismos lingüísticos que, en las cuatro lenguas romances, permiten producir determinados efectos de sentido en los interlocutores. El propósito de las actividades con el lenguaje es que los adolescentes puedan involucrarse como sujetos críticos en problemas sociales y desarrollar habilidades como hablantes competentes de su lengua mediante los procesos de intercomprensión. En ese sentido, entendemos que las problemáticas ambientales conforman un contexto cognitivo donde cobra sentido la lectura en diferentes lenguas. Los textos escritos en francés, italiano y portugués permiten atender el modo de enfocar temas ambientales en otros contextos culturales. Con ello, la perspectiva de aprendizaje de las lenguas emparentadas se ve enriquecida.

Es importante destacar que en cada secuencia didáctica se promueve el desarrollo de estrategias de intercomprensión en las cuatro lenguas romances y de habilidades de producción escrita en español. Desde nuestra perspectiva, la comprensión de textos en las distintas lenguas y el análisis contrastivo en el nivel de los procedimientos de enunciación, los géneros textuales y los mecanismos lingüísticos de cada idioma son procesos que favorecen el desarrollo de la escritura de textos en lengua materna. Por otra parte, en cada secuencia didáctica proponemos estrategias de intercomprensión variadas, que respetan una progresión en las habilidades.

Así, por ejemplo, en la primera secuencia, que aborda el artículo enciclopédico, dichas estrategias están orientadas a (1) activar saberes sobre los parámetros del contexto de producción a través de preguntas que apelan a las experiencias con el género y a la consideración de aspectos del contexto socio-subjetivo (intencionalidades del emisor y del receptor, roles, etc.); (2) identificar el contenido temático de cada texto; (3) seleccionar y jerarquizar contenido relevante sobre el yaguareté para integrarlo y organizarlo en un esquema, tarea concomitante con la planificación de la escritura. 
Figura 4 - Actividades propuestas a partir de la lectura de artículos enciclopédicos escritos en las cuatro lenguas

Después de la lectura respondé a las siguientes preguntas:

1. En tu opinión, ¿QQuién o quiénes son los lectores eventuales de estos textos?

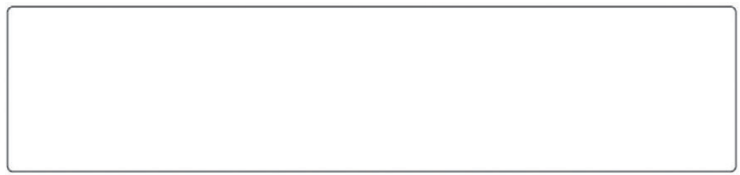

2. Todo texto cumple una función comunicativa, se dice algo a alguien con una determinada intención ¿̨Cuál creés que es la intención o finalidad comunicativa de los autores de estos articulos? ¿Qué efecto buscan producir en esos lectores eventuales?

3. ¿Qué información esencial sobre el jaguar proporciona cada uno de los textos? Escribí en pocas lineas el tema de cada texto

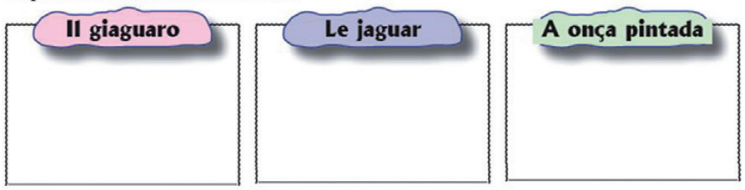

5. Completá el siguiente organizador gráfico con la información que nos proporcionan los cuatro textos sobre el yaguareté.

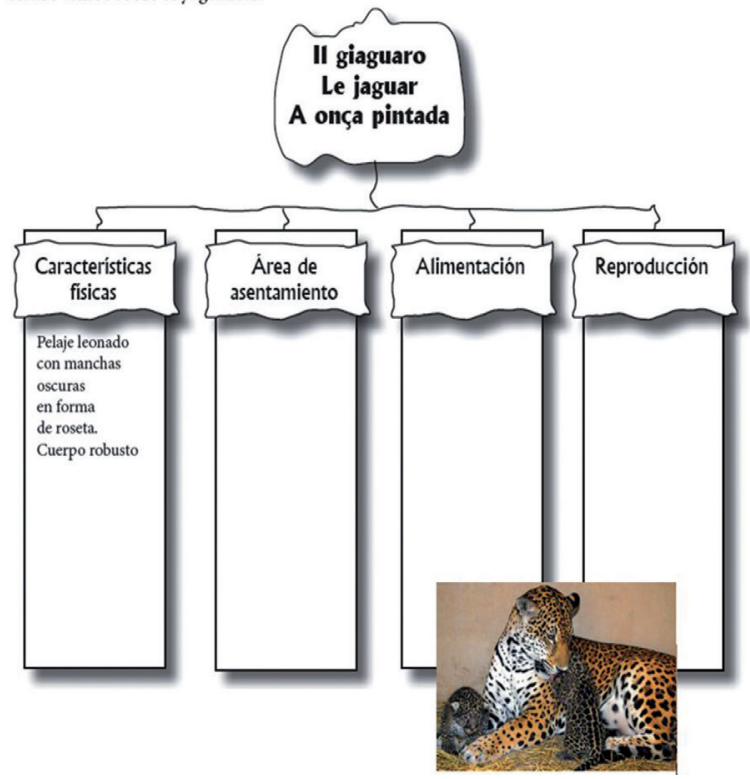

Fuente: Elaboración propia. 
Además, en esta secuencia didáctica tiene un espacio significativo al tratamiento del léxico. Como se sabe, la lectura de los artículos enciclopédicos requiere del dominio del vocabulario de las ciencias, que conforma redes jerárquicas donde cada término se relaciona con otros a partir de categorizaciones lingüísticas basadas en procesos de generalización y de abstracción. Al respecto, es preciso recordar la diferenciación que propone Vygotsky (1962) entre conceptos espontáneos y conceptos científicos, enfoque que es fundamental para la comprensión de la prosa científica. Desde su perspectiva, ambos tipos de conceptos se diferencian a partir de sus características y del modo de adquisición. Básicamente, mientras los conceptos cotidianos se desarrollan espontáneamente, en la interacción social, los conceptos científicos solo se adquieren a partir de la enseńanza sistemática y deliberada.

Además, estos últimos son el resultado de procesos de reflexión y de sistematización. En el caso de los artículos enciclopédicos sobre el yaguareté en las tres lenguas romances, se pueden señalar, entre otros, los siguientes conceptos: predatore, individui, aspettativa di vita (it.); carnivoro, gestação (port.); mammifêre carnivore, félidés, espèce (fr.). Normalmente, para comprender el significado de estas expresiones es preciso acceder a las redes conceptuales que las incluyen, cuyo conocimiento se adquiere mediante una enseñanza sistemática. Por ejemplo, la noción de superpredador supone conocimientos previos construidos por la ciencia occidental en torno de la depredación como una forma de interacción entre poblaciones, donde una especie se beneficia - depredador- y otra se perjudica -presa(CURTIS et al., 2011). En ese contexto conceptual se denomina superpredador a aquel animal carnívoro que prevalece sobre las otras especies en un ecosistema, es decir, que no puede ser presa de otro animal, con lo cual se ubica en la cima de la cadena alimentaria. Por lo tanto, si conocemos el concepto de superpredador, al leer la siguiente oración: "C'est aussi un superprédateur qui joue un rôle important dans la stabilisation des écosystèmes et la régulation des populations qu'il chasse” (Figura 5), podremos saber, en principio, que el yaguareté se alimenta de distintas presas pero nunca será cazado por otros animales. 
Figura 5 - Texto sobre el yaguareté incluido en la primera secuencia didáctica

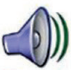

\section{Le jaguar}

Espèce quasi menacée selon l'Union Internationale pour la Conservation de la Nature, le jaguar est un mammifere carnivore de la famille des félidés. C'est l'un des quatre "grands félins" du genre Panthera, avec le tigre, le lion et le léopard. Son aire de répartition actuelle s'étend du Mexique à la majeure partie de l'Amérique Centrale et de l'Amérique du Sud, jusqu'au nord de l'Argentine et du Paraguay.

Ce félin tacheté ressemble physiquement au léopard, mais il est généralement plus grand et d'apparence plus massive. En outre son habitat et son comportement sont plus proches de ceux du tigre. Bien que la forêt tropicale dense constitue son habitat de prédilection, le jaguar s'accommode d'une large variété de milieux ouverts et boisés. Il est fortement associé à la présence de l'eau et, comme le tigre, il aime nager. C'est un prédateur solitaire qui chasse à l'affût, tout en étant opportuniste dans la sélection des proies. C'est aussi un superprédateur qui joue un rôle important dans la stabilisation des écosystèmes et la régulation des populations qu'il chasse.

HABITAT

Le Pantanal au Brésil: zone humide appréciée du jaguar.

L'habitat du félin comprend les forêts tropicales de l'Amérique Centrale et du Sud qui sont saisonnièrement inondées. La présence d'eau (marécages, lacs, mangroves) est un paramètre important de son installation dans une région.

Texto completo en: http:/fr.wikipedia.org/wiki/Jaguar\#Habitat

Fuente: JAGUAR... (2015).

En las cuatro lenguas romances estudiadas se puede reconocer modos similares de utilizar conceptos científicos, más allá del parecido que puedan tener las formas léxicas: felinos, felinos, felini, félins; especie, espécie, specie, espèce; presa, presa, prede, proie. Es común a estas lenguas, por ejemplo, la taxonomía que propone la ciencia. Así, cuando leemos mamifero, mamifero, mammifero, mammifere, nuestra memoria operativa activa una red jerárquica de conceptos donde ese término es solidario con anfibio, reptil, pez y ave. A su vez, estas expresiones, que están integradas en el concepto de vertebrados, incluyen conceptos más específicos. Por ejemplo, la expresión mamífero abarca a carnívoros, primates, etc., que el sistema jerárquico de clasificación biológica agrupa en la categoría orden (ver Figura 6). 
Figura 6 - Sistema de clasificación biológica

basado en los niveles propuestos por Linneo

\begin{tabular}{|c|c|c|c|c|c|}
\hline Reino & & Animales (vertebrados) \\
\hline Clase & reptiles & mamiferos & peces & anfibios & aves \\
\hline Orden & & carnivoros & primates & pinipedos & cetáceos \\
\hline Familia & canidae & mustelidae & felidae & procyonidae \\
\hline Género & & panthera & & \\
\hline
\end{tabular}

Fuente: Elaboración propia a partir de la información de Canevari y Fernández Balboa (2003).

El conocimiento de las redes conceptuales relacionadas, en este caso, con la clasificación de los animales vertebrados es guiado en la primera secuencia didáctica mediante actividades que promueven la adquisición de conceptos de las ciencias naturales, el aprendizaje de las lenguas y, con ello, el desarrollo de la cognición. A modo de ejemplo, la Figura 7 presenta una actividad de descubrimiento de una expresión intrusa. Para resolver esta tarea, los alumnos deberán relacionar la imagen con el nombre del animal en las distintas lenguas, comparar los animales, identificar rasgos comunes entre ellos, reconocer la expresión que los incluye y, por último, señalar la especie que queda fuera de ese concepto.

De ese modo, le lion comparte con le jaguar, le léopard, le chat y le tigre las características que los incluye en la denominación de felinos o félins (Familia Felidae). A su vez, o leão, a hiena, o crocodilo, o falcão y o tubarão forman parte del orden de os carnivoros, con lo cual o boi es la expresión intrusa. En la serie 3, il leone, la giraffa, la balena, il cavallo y l'orso pertenecen a la clase mammifero, por lo tanto, la tartaruga queda excluida de ese grupo. 
Figura 7 - Actividad para desarrollar el léxico vinculado con el artículo enciclopédico

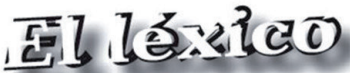

2. Intrusos. Descubrí la palabra que no pertenece al grupo y justificá tu respuesta.

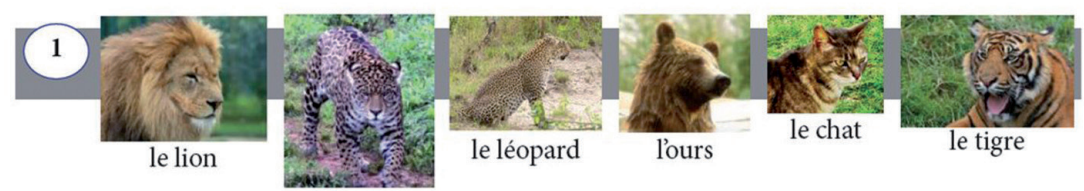

le jaguar
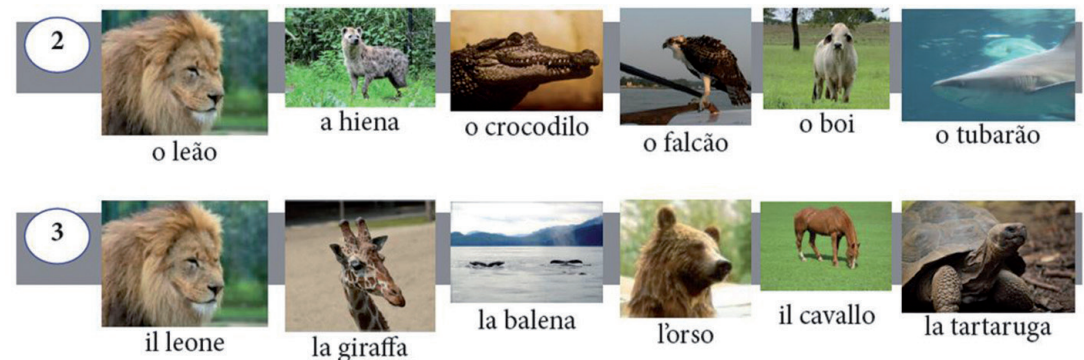

Fuente: Elaboración propia.

Como se advierte, esta actividad promueve la adquisición de criterios de clasificación de la biología, basados en operaciones de abstracción y generalización, y el desarrollo del vocabulario no solo en lengua materna sino en las otras tres lenguas romances. Además, motiva la posibilidad de reflexionar de modo contrastivo sobre las correspondencias en el léxico panrománico, el género de los sustantivos que designan animales en las distintas lenguas, etc. En ese sentido, es posible sostener que las actividades de trabajo con el léxico mediadas por estrategias de intercomprensión promueven una reflexión más profunda sobre los procedimientos de designación en lengua materna. Por último, atendiendo al procesamiento de la información textual, entendemos que este trabajo con el vocabulario facilita la realización de inferencias léxicas vinculadas con los conceptos científicos que contribuyen a una comprensión adecuada de los textos.

\section{Conclusiones}

Desde la perspectiva de nuestro equipo es necesario promover propuestas de educación lingüística que entiendan el aprendizaje como una construcción conjunta y activa de los conocimientos, donde la lengua es mediadora de procesos de reflexión y de acción. Abordar los temas ambientales a partir géneros textuales en diversas lenguas nos acerca a temas de la diversidad cultural, promueve el reconocimiento y 
el respeto de visiones e intereses en relación con el ambiente en el marco semiótico de una lengua determinada, que utiliza una materia expresiva específica y se organiza en paradigmas léxicos y en estructuras morfosintácticas que dan cuenta de las relaciones entre un agente enunciador con el contexto de acción, en este caso, el contexto ecológico. Desde esta perspectiva, al entrar en contacto con la diversidad lingüística, la intercomprensión contribuye al desarrollo de una ecología de las lenguas en el marco de actividades didácticas que revalorizan las relaciones de las lenguas entre sí y de las lenguas con su entorno.

\section{THE DEVELOPMENT OF ENVIRONMENTAL AWARENESS THROUGH A PROPOSAL FOR PROMOTING INTERCOMPREHENSION IN ROMANCE LANGUAGES AMONG HIGH SCHOOL SENIORS}

- ABSTRACT: This work aims to analyze the treatment of environmental issues in an educational proposal for Intercomprehension in Romance Languages (hereinafter called IC) developed by InterRom, a research team from Universidad Nacional de Córdoba, in Argentina. This proposal, aimed at developing high school seniors' language skills from the perspective of teaching and learning IC from text genres, integrates pluralistic approaches to languages with contributions from socio-discursive interactionism (BRONCKART, 2007), from the sociocultural theory of human development (BRUNER, 1994; NELSON, 1996; ROGOFF, 1993; VYGOTSKY, 1962, 1988) and from cognitive approaches to text comprehension and production (BRITTON and GRAESSER, 1996; HAYES and FLOWERS, 1980, HAYES, 1996; SCARDAMALIA and BEREITER, 1992). Through the convergence of these theoretical perspectives, the proposal promotes IC in French, Italian, Spanish and Portuguese, related to environmental conservation, as well as actions for writing texts in Spanish. The planned language activities involve working with different text genres on environmental issues, such as the extinction of animal species, the production of genetically modified foods and the use of agrochemicals that contaminate the biosphere and threaten the life of all living species, including human beings. The exercises related to these topics, at the same time, encourage students to reflect on critical environmental issues and to develop strategies for writing texts in Spanish. To conclude, it can be argued that the IC proposal is based on social and intercultural approaches that integrate teaching actions, text production processes and science issues regarding the environment. As a result, students are regarded as subjects with competencies to understand discourses from different cultures and to think, write and act within their natural, social and cultural environments.

- KEYWORDS: Plurilingualism. Intercomprehension. Text genres. Environment 


\section{REFERÊNCIAS}

ADAM, J. M. (Proto)Tipos: la estructura de la composición en los textos. Revista Textos de Didáctica de la Lengua y de la Literatura, Barcelona, n.10, p.9-22, 1996.

AGUILAR, Z. et al. Bases para la conservación de la biodiversidad y herramientas de manejo y uso sostenible: programa para la gestión democrática de los recursos naturales. Quito: EcoCiencia, 2006.

ARGENTINA. Ley n. 26.206, de 14 diciembre de 2006. Ley de Educación Nacional. Argentina, 27 dic. 2006. Disponible en: <http://portal.educacion.gov.ar/consejo/files/2009/12/ ley_de_educ_nac1.pdf>. Acceso en: 01 jun. 2015.

BAJTIN, M. Estética de la creación verbal. México: Siglo XXI, 1982.

BRITTON, B.; GRAESSER, A. Models of understanding text. New Jersey: Lawrence Erlbaum Associates, 1996.

BRONCKART, J. P. Desarrollo del lenguaje y didáctica de las lenguas. Buenos Aires: Miño \& Dávila, 2007.

BRUNER, J. Acción, pensamiento y lenguaje. Madrid: Alianza. 1994.

CAMPS, A. Modelos del proceso de redacción: algunas implicancias para la enseñanza. Infancia y Aprendizaje: Journal for the Study of Education and Development, [S.1.], n.49, p.3-19, 1990. Disponible en: <http://dialnet.unirioja.es/servlet/articulo? $\operatorname{codigo}=48341>$. Acceso en: 20 maio 2015.

CANDELIER, M. (Coord.). CARAP: cadre de référence pour les approches plurielles des langues et des cultures. Strasbourg: Centre européen pour les Langues Vivantes/ Conseil de l'Europe, 2008. Disponible en: <http://carap.ecml.at/Portals/11/documents/MAREP_Cast_ vers5def_08.pdf>. Acceso en: 20 maio 2015.

CANEVARI, M.; FERNÁNDEZ BALBOA, C. Cien mamíferos argentinos. Buenos Aires: Albatros, 2003.

CORRADINI, N. La mostra sul popolo indio Kayapo. 2003. Disponible en: <http://www. saveriani.it/comunita/salerno/Notizie/2003/01.htm>. Acceso en: 15 jun. 2015.

CURTIS, H. et al. Biología. 7.ed. Buenos Aires: Médica Panamericana, 2011.

GASCHÉ, J. Niños, maestros, comuneros y escritos antropológicos como fuentes de contenidos indígenas escolares y la actividad como punto de partida de los procesos pedagógicos interculturales: un modelo sintáctico de cultura. Red Internacional de Estudios Interculturales, Perú, p.01-68, 2004. Disponible en: <http://red.pucp.edu.pe/ridei/wpcontent/uploads/biblioteca/ninos_maestros_comuneros_modelo_sintactico_de_cultura.pdf $>$. Acceso en: 20 maio 2015. 
GUREVICH, R. (Comp.). Ambiente y educación: una apuesta al futuro. Buenos Aires: Paidós, 2011.

HALLIDAY, M. A. K. Towards a language-based theory of learning. Linguistics and Education, New York, v.5, n.2, p.93-31, 1993. Disponible en: <http://lchc.ucsd.edu/mca/ Paper/JuneJuly05/HallidayLangBased.pdf>. Acceso en: 20 maio 2015.

HAYES, J.; FLOWER, L. Identifying the organization of writing processes. En: GREGG, L. W.; STEINNBERG, E. R. (Ed.). Cognitive processes in writing. Hillsdale: Erlbaum, 1980. $\mathrm{p}-3-30$.

HAYES, J. R. A new framework for understanding cognition and affect in writing. En: LEVY, C.; RANSDELL, S. (Ed.). The science of writing: theories, methods, individual differences, and applications. Mahwah: Erlbaum, 1996. p.1-27.

JAGUAR: Panthera onca. In: WIKIPÉDIA l'encyclopeédie libre. Disponible en: <http:// fr.wikipedia.org/wiki/Jaguar>. Acceso en: 15 jun. 2015.

NELSON, K. Language in cognitive development: the emergence of the mediated mind. Cambridge: Cambridge University Press, 1996.

ROGOFF, B. Aprendices del pensamiento: el desarrollo cognitivo en el contexto social. Barcelona: Paidós, 1993.

SCARDAMALIA, M.; BEREITER, C. Dos modelos explicativos de los procesos de composición escrita. Infancia y Aprendizaje, Madrid, n.58, p.43-64, 1992.

SILVESTRI, A. Los géneros discursivos y el desarrollo del pensamiento: un enfoque sociocultural. En: DUBROVSKY, S. (Comp.). Vygotsky: su proyección en el pensamiento actual. Buenos Aires: Novedades Educativas, 2000. p.87-95.

. Bajtín y Vygotsky: teoría del enunciado y concepción socio-cultural del psiquismo. En: ROSA, A.; VALSINER, J. Historical and theoretical discourse. Madrid: Fundación Infancia y Aprendizaje, 1994. p.213-221.

SURVIVAL. Nueva oleada de protestas indígenas en la Amazonia contra la construcción de presas. Madrid, 27 out. 2009. Disponible en: <http://www.survival.es/noticias/5163>. Acceso en: 16 jun. 2015

VYGOTSKY, L. S. El desarrollo de los procesos psicológicos superiores. Barcelona: Grijalbo, 1988.

Pensamiento y lenguaje. Buenos Aires: Lautaro, 1962. 
